\title{
KINETICS OF THE STOCHASTIC ISING CHAIN IN A TWO-FLIP MODEL
}

\author{
H. J. HILHORST, M. SUZUKI ${ }^{\ddagger}$ and B. U. FELDERHOF \\ Instituut voor Theoretische Fysica der Rijksuniversiteit, \\ Utrecht, Nederland
}

Received 5 October 1971

\section{Synopsis}

The exact solution is presented of a stochastic model for the cyclic Ising chain of $N$ spins $\frac{1}{2}$ with nearest-neighbour interactions in the absence of a magnetic field. In each transition two neighbouring spins simultaneously change their states with transition probability determined by the state of their neighbours and the temperature of a heat bath. A set of spin operators is constructed whose average values decay to their equilibrium values exponentially in time. Relaxation of the Fourier components of magnetization and energy density is treated in detail.

1. Introduction. The study of stochastic Ising spin systems in interaction with a heat bath was initiated by Glauber ${ }^{1}$ ) in 1963 . He proposed a model in which each spin individually can change its state with transition probability determined by the states of its neighbours, by the temperature of a heat bath, and by the external magnetic field ${ }^{2}$ ). The model is of relevance for the theory of critical slowing down. For the 2- and 3-dimensional Ising model critical exponents were determined numerically by Yahata and Suzuki ${ }^{3}$ ). Glauber showed that for the Ising chain with nearest-neighbour interactions in the absence of a magnetic field the equations for the average values of individual spin variables and of products of two spin variables can be solved exactly. Bedeaux, Shuler and Oppenheim ${ }^{4}$ ) solved the hierarchy of moment equations for averages of products of any number of spin variables. An alternative complete solution of the master equation for this model was given by Felderhof ${ }^{5}$ ).

Recently two of us (B.U.F. and M.S.) considered again the Ising chain but changed the kinetics of the model ${ }^{6}$ ). It was shown that if in each transition $n$ successive spins change their states the time-dependent statistics of bulk properties of the system can be studied exactly in the limit of an infinitely long chain, i.e. neglecting boundary effects. In this limit the equilibrium time correlation functions

\footnotetext{
₹ Present address: Institute for Solid State Physics, University of Tokyo, Tokyo, Japan.
} 
of Fourier components of energy density and magnetization were evaluated. In the present paper we consider the particular case of the 2-flip model, in which pairs of neighbouring spins change state in a transition. It is shown that for this case the master equation may be solved exactly even for a finite chain. The same methods are used as in ref. 5 (hereafter referred to as I). Since in this case the equations for the averages of products of spin variables do not decouple as in the 1-flip model, it is of interest to see how our solution leads naturally to the linear combinations with simple exponential behaviour in time.

As in the case of the 1-flip model an exact solution of the 2-flip model is possible only when the rates of energy conserving and energy nonconserving transitions are related in a specific manner. This limits the applicability of the model in practical situations, but we feel that nevertheless an exact solution is worthwhile since it enables one to test approximate methods designed for use in a wider range of circumstances.

2. Master equation of the two-flip model. We consider a cyclic Ising chain of $N$ spins $\frac{1}{2}$. The configurations of the chain will be denoted by $s_{N} \equiv\left(s_{1}, s_{2}, \ldots, s_{N}\right)$ where the variables $s_{f}$ take the values \pm 1 , corresponding to spin up or down. The energy of a spin configuration in the absence of a magnetic field is given by

$$
E\left(s_{N}\right)=-J \sum_{j=1}^{N} s_{j} s_{j+1},
$$

where $s_{N+1} \equiv s_{1}$. In the kinetic Ising model the spins are assumed to flip stochastically due to the interactions with a heat bath of temperature $T$. The probability distribution $P\left(s_{N}, t\right)$ satisfies a master equation

$$
\frac{\partial P\left(s_{N}, t\right)}{\partial t}=\sum_{s_{N}^{\prime}}\left[W\left(s_{N}, s_{N}^{\prime}\right) P\left(s_{N}^{\prime}, t\right)-W\left(s_{N}^{\prime}, s_{N}\right) P\left(s_{N}, t\right)\right] .
$$

In the 2-flip model the transition probabilities are given by

$$
\begin{aligned}
W\left(s_{N}^{\prime}, s_{N}\right)= & \frac{1}{2} \alpha \sum_{j=1}^{N}\left[1-\frac{1}{2} \gamma\left(s_{j-1} s_{j}+s_{j+1} s_{j+2}\right)\right] \delta\left(\left|s_{j}-s_{j}^{\prime}\right|-2\right) \\
& \times \delta\left(\left|s_{j+1}-s_{j+1}^{\prime}\right|-2\right) \prod_{k \neq j, j+1} \delta\left(s_{k}-s_{k}^{\prime}\right),
\end{aligned}
$$

where $\alpha$ is a rate constant and $\gamma$ is found from a detailed-balance consideration to be given by

$$
\gamma=\tanh 2 \beta J
$$

with $\beta=1 / k_{B} T$. In ref. 6 we have discussed how a realistic interaction with a heat bath leads to a temperature-dependent $\alpha$. Expression (2.3) is not the most general 
possible but involves a particular relation between the rates of energy conserving and energy nonconserving transitions which is necessary to make the model solvable.

Since in each transition two spins simultaneously change their states the configurations $s_{N}$ may be divided into two classes which are not connected by transitions, each class being characterized by the sign of the product variable

$$
\pi_{z}\left(s_{N}\right)=\prod_{j=1}^{N} s_{j} .
$$

The relative weight of each class is conserved in the course of time and may be expressed in terms of a parameter $\mu$ with values between -1 and +1 , defined by

$$
\mu=\sum_{s_{N}} \pi_{z}\left(s_{N}\right) P\left(s_{N}, t\right)
$$

As $t$ tends to infinity the probability distribution tends to the equilibrium distribution

$$
P_{\text {eq, }}\left(s_{N}\right)=\frac{1}{2}(1+\mu) P_{\text {eq, }}\left(s_{N}\right)+\frac{1}{2}(1-\mu) P_{\text {eq, }}\left(s_{N}\right),
$$

where

$$
P_{\text {eq, } \pm}\left(s_{N}\right)=\frac{1}{2}\left[1 \pm \pi_{z}\left(s_{N}\right)\right] \exp \left[-\beta E\left(s_{N}\right)\right] / Z_{N_{ \pm}} \cdot
$$

The partition functions $Z_{N \pm}$ are easily evaluated by the transfer-matrix method and one finds

$$
Z_{N \pm}=\frac{1}{2}[2 \cosh K]^{N}\left\{1+a^{N} \pm\left[1+(-1)^{N}\right] a^{\frac{1}{2} N}\right\}
$$

where $K=\beta J$ and $a=\tanh K$.

As in I it is convenient to represent a configuration $s_{N}$ as the direct product of $N$ Pauli spinors. The direct product will be denoted by $\left|s_{N}\right\rangle$ and will be regarded as a basis vector in a $2^{N}$-dimensional complex vector space $\mathfrak{E}_{N}$ endowed with the usual scalar product. The basis vectors $\left|s_{N}\right\rangle$ satisfy the orthonormality relation

$$
\left\langle s_{N} \mid s_{N}^{\prime}\right\rangle=\delta\left(s_{N}-s_{N}^{\prime}\right)
$$

where $\delta\left(s_{N}-s_{N}^{\prime}\right)$ is shorthand for a product of $N$ Kronecker deltas. The probability distribution $P\left(s_{N}, t\right)$ may be represented uniquely by a time-dependent vector in $\mathfrak{E}_{N}$,

$$
|P(t)\rangle=\sum_{s_{N}} P\left(s_{N}, t\right)\left|s_{N}\right\rangle
$$


The master equation (2.2) corresponds to the equation of motion

$$
\frac{\partial}{\partial t}|P(t)\rangle=\mathscr{W}|P(t)\rangle
$$

where the linear operator $\mathscr{W}$ is given by

$$
\begin{aligned}
\mathscr{W}= & \frac{1}{2} \alpha \sum_{j=1}^{N}\left\{\left[1+\frac{1}{2} \gamma\left(\sigma_{j-1}^{z} \sigma_{j}^{z}+\sigma_{j+1}^{z} \sigma_{j+2}^{z}\right)\right] \sigma_{j}^{x} \sigma_{j+1}^{x}\right. \\
& \left.-\left[1-\frac{1}{2} \gamma\left(\sigma_{j-1}^{z} \sigma_{j}^{z}+\sigma_{j+1}^{z} \sigma_{j+2}^{z}\right)\right]\right\} .
\end{aligned}
$$

The operators $\sigma_{j}^{x}$ and $\sigma_{j}^{z}$ act as Pauli spin operators on the $j$ th spinor and as unit operators on all the others. For $j=0$ and $j>N$ we use a cyclic definition. The master operator $\mathscr{W}$ commutes with the sign operator

$$
\Pi^{z}=\prod_{j=1}^{N} \sigma_{j}^{z}, \quad \Pi^{z}\left|s_{N}\right\rangle=\pi_{z}\left(s_{N}\right)\left|s_{N}\right\rangle
$$

which shows again that $\mu$ is conserved. The sign operator divides $\mathfrak{\mathfrak { g }}_{N}$ into two subspaces $\mathfrak{G}_{N, \pm}$ which are invariant under the operator $\mathscr{W}$. The corresponding projection operators are

$$
\mathscr{P}_{ \pm}=\frac{1}{2}\left(1 \pm \Pi^{z}\right)
$$

The unit state $|1\rangle$ is defined by

$$
|1\rangle=\sum_{s_{N}}\left|s_{N}\right\rangle=\left(\begin{array}{l}
1 \\
1
\end{array}\right)_{1} \times\left(\begin{array}{l}
1 \\
1
\end{array}\right)_{2} \times \cdots \times\left(\begin{array}{l}
1 \\
1
\end{array}\right)_{N} .
$$

The norm of this state is $\langle 1 \mid 1\rangle=2^{N}$ and normalization of the probability distribution $P\left(s_{N}, t\right)$ is expressed by

$$
\langle 1 \mid P(t)\rangle=1 .
$$

It is convenient to introduce similar unit states for each of the subspaces $\mathfrak{S}_{N, \pm}$,

$$
\left|1_{ \pm}\right\rangle=\mathscr{P}_{ \pm}|1\rangle
$$

with norm

$$
\left\langle 1_{ \pm} \mid 1_{ \pm}\right\rangle=2^{N-1}
$$

We also define

$$
\left|P_{ \pm}(t)\right\rangle=\mathscr{P}_{ \pm}|P(t)\rangle,
$$


with the normalization

$$
\left\langle 1_{ \pm} \mid P_{ \pm}(t)\right\rangle=\frac{1}{2}(1 \pm \mu) .
$$

Each of the vectors $\left|P_{+}(t)\right\rangle$ and $\left|P_{-}(t)\right\rangle$ separately satisfies the master equation (2.12). In particular

$$
\mathscr{W}\left|P_{\text {eq }, \pm}\right\rangle=0
$$

i.e. the equilibrium vectors $\left|\boldsymbol{P}_{\mathrm{eq}, \pm}\right\rangle$ both are eigenvectors of the master operator with eigenvalue zero. For the explicit solution of the master equation we shall find the complete set of eigenvectors and eigenvalues.

3. Diagonalization of the master operator. For the explicit diagonalization it is convenient to symmetrize the master operator by means of the transformation

$$
\mathscr{W}(\beta)=\varrho_{\mathrm{eq}}^{-\frac{1}{2}} \mathscr{W} \varrho_{\mathrm{eq}}^{\frac{1}{2}},
$$

where

$$
\begin{aligned}
& \varrho_{\text {eq }}=\exp \left(-\beta \mathscr{H}_{N}\right) / Z_{N}, \\
& \mathscr{H}_{N}=-J \sum_{j=1}^{N} \sigma_{j}^{z} \sigma_{j+1}^{z}, \\
& Z_{N}=Z_{N+}+Z_{N-\cdot}
\end{aligned}
$$

The similarity transformation (3.1) may be applied to any linear operator acting in $\mathfrak{E}_{N}$. As in I we have

$$
\begin{aligned}
\sigma_{J}^{z}(\beta)= & \sigma_{j}^{z}, \\
\sigma_{j}^{x}(\beta)= & \sigma_{j}^{x}\left[\cosh ^{2} K+\sigma_{j-1}^{z} \sigma_{j+1}^{z} \sinh ^{2} K\right] \\
& -\mathrm{i} \sigma_{j}^{y}\left[\sigma_{j-1}^{z}+\sigma_{j+1}^{z}\right] \sinh K \cosh K .
\end{aligned}
$$

Substituting one finds

$$
\begin{aligned}
\mathscr{W}(\beta)= & \frac{1}{2} \alpha \sum_{j=1}^{N}\left\{\left[\cos ^{2} \varphi \sigma_{j}^{x} \sigma_{j+1}^{x}+\sin ^{2} \varphi \sigma_{j-1}^{z} \sigma_{j}^{y} \sigma_{j+1}^{y} \sigma_{j+2}^{z}\right]\right. \\
& \left.-\left[1-\frac{1}{2} \gamma\left(\sigma_{j-1}^{z} \sigma_{j}^{z}+\sigma_{j+1}^{z} \sigma_{j+2}^{z}\right)\right]\right\}
\end{aligned}
$$

where

$$
\begin{aligned}
& \cos \varphi=\cosh K /\left[\cosh ^{2} K+\sinh ^{2} K\right]^{\frac{1}{2}}, \\
& \sin \varphi=\sinh K /\left[\cosh ^{2} K+\sinh ^{2} K\right]^{\frac{1}{2}}, \\
& \gamma=\tanh 2 K=\sin 2 p .
\end{aligned}
$$


A corresponding transformation is applied to the vectors of $\mathfrak{S}_{N}$. In particular we define

$$
|\psi(t)\rangle=\varrho_{\mathrm{eq}}^{-\frac{1}{2}}|P(t)\rangle .
$$

The density operator $\varrho_{\text {eq }}$ commutes with $\Pi^{z}$ so that the subspaces $\mathscr{H}_{N, \pm}$ are invariant under the transformation. The equilibrium vectors transform to

$$
\left|0_{ \pm}\right\rangle=\varrho_{\mathrm{eq}}^{-\frac{1}{2}}\left|P_{\mathrm{eq}, \pm}\right\rangle=\left(Z_{N} / Z_{N_{ \pm}}\right) \varrho_{\mathrm{eq}}^{\frac{1}{2}}\left|1_{ \pm}\right\rangle .
$$

Hence the two "vacuum" states $\left|0_{ \pm}\right\rangle$are normalized to

$$
\left\langle 0_{ \pm} \mid 0_{ \pm}\right\rangle=Z_{N} / Z_{N_{ \pm}} \text {. }
$$

The master equation (2.12) is transformed to

$$
\frac{\partial}{\partial t}|\psi(t)\rangle=\mathscr{W}(\beta)|\psi(t)\rangle
$$

and (2.22) becomes

$$
\mathscr{W}(\beta)\left|0_{ \pm}\right\rangle=0 .
$$

For the explicit diagonalization of $\mathscr{W}(\beta)$ we apply the following Jordan-Wigner transformation

$$
\begin{aligned}
& a_{n}^{\dagger}=\frac{1}{2} \prod_{j=1}^{n-1} \sigma_{j}^{y}\left(\sigma_{n}^{x}-\mathrm{i} \sigma_{n}^{z}\right), \\
& a_{n}=\frac{1}{2} \prod_{j=1}^{n-1} \sigma_{j}^{y}\left(\sigma_{n}^{x}+\mathrm{i} \sigma_{n}^{z}\right) .
\end{aligned}
$$

It is easily verified that the operators $a_{n}^{\dagger}$ and $a_{n}$ satisfy the fermion anticommutation rules. The inverse transformation reads

$$
\begin{aligned}
& \sigma_{n}^{x}=\prod_{j=1}^{n-1}\left(2 a_{j}^{\dagger} a_{j}-1\right)\left(a_{n}^{\dagger}+a_{n}\right), \\
& \sigma_{n}^{y}=2 a_{n}^{\dagger} a_{n}-1, \\
& \sigma_{n}^{z}=\mathrm{i} \prod_{j=1}^{n-1}\left(2 a_{j}^{\dagger} a_{j}-1\right)\left(a_{n}^{\dagger}-a_{n}\right) .
\end{aligned}
$$

The products of Pauli spin operators occurring in $\mathscr{W}(\beta)$ read in terms of fermion operators

$$
\begin{aligned}
& \sigma_{j}^{x} \sigma_{j+1}^{x}=-\left(a_{j}^{\dagger}-a_{j}\right)\left(a_{j+1}^{\dagger}+a_{j+1}\right) \quad(j \neq N), \\
& \sigma_{j}^{z} \sigma_{j+1}^{z}=\left(a_{j}^{\dagger}+a_{j}\right)\left(a_{j+1}^{\dagger}-a_{j+1}\right) \quad(j \neq N), \\
& \sigma_{j}^{z} \sigma_{j+1}^{y} \sigma_{j+2}^{y} \sigma_{j+3}^{z}=\left(a_{j}^{\dagger}+a_{j}\right)\left(a_{j+3}^{\dagger}-a_{j+3}\right) \quad(j \neq N-2, N-1, N) .
\end{aligned}
$$


The boundary terms read for $N$ even

$$
\begin{aligned}
& \sigma_{N}^{x} \sigma_{1}^{x}=(-1)^{\mathscr{N}_{a}}\left(a_{N}^{\dagger}-a_{N}\right)\left(a_{1}^{\dagger}+a_{1}\right), \\
& \sigma_{N}^{z} \sigma_{1}^{z}=-(-1)^{\mathscr{N}_{a}}\left(a_{N}^{\dagger}+a_{N}\right)\left(a_{1}^{\dagger}-a_{1}\right), \\
& \sigma_{f}^{z} \sigma_{J+1}^{y} \sigma_{J+2}^{y} \sigma_{J+3}^{z} \\
& =-(-1)^{\mathscr{V}_{a}}\left(a_{j}^{\dagger}+a_{j}\right)\left(a_{j+3}^{\dagger}-a_{j+3}\right) \quad(j=N-2, N-1, N),
\end{aligned}
$$

where

$$
\mathcal{N}_{a}=\sum_{j=1}^{N} a_{j}^{\dagger} a_{j},
$$

is the operator for the total number of $a$ particles. Evidently, apart from some boundary terms, the master operator $\mathscr{W}(\beta)$ is bilinear in the fermion operators and can be diagonalized in the usual manner. Before proceeding, however, it is expedient to define new fermion operators with properties that simplify later calculations. We define

$$
c_{n}=\frac{1}{2}\left(a_{n+1}^{\dagger}+a_{n+1}-a_{n}^{\dagger}+a_{n}\right) \quad(n=1, \ldots, N),
$$

where $a_{N+1} \equiv a_{1}$. Consequently, from (3.11),

$$
\begin{aligned}
& c_{n}=\frac{1}{2}\left(\prod_{j=1}^{n} \sigma_{j}^{y}\right)\left(-\sigma_{n}^{x}+\sigma_{n+1}^{x}\right) \quad(n=1, \ldots, N-1), \\
& c_{N}=\frac{1}{2}\left[-\left(\prod_{j=1}^{N} \sigma_{j}^{y}\right) \sigma_{N}^{x}+\sigma_{1}^{x}\right] .
\end{aligned}
$$

These operators have the advantage that their action on the unit states $\left|1_{ \pm}\right\rangle$is relatively simple, viz.,

$$
\begin{aligned}
& c_{n}\left|1_{ \pm}\right\rangle=0 \quad(n=1, \ldots, N-1), \\
& c_{N}\left|1_{-}\right\rangle=0, \quad c_{N}^{\dagger}\left|1_{+}\right\rangle=0 .
\end{aligned}
$$

Here and in the following we choose $N$ to be a multiple of 4 . Although the expressions for the spin operators in terms of $c$ operators are complicated, the combinations which are needed in $\mathscr{W}(\beta)$ are again simple and are easily found from the relations

$$
\begin{aligned}
& c_{n}^{\dagger}-c_{n}=a_{n}^{\dagger}-a_{n}, \\
& c_{n}^{\dagger}+c_{n}=a_{n+1}^{\dagger}+a_{n+1} \quad(n=1, \ldots, N), \\
& (-1)^{\mathscr{N}_{c}}=-(-1)^{\mathscr{N}_{a}} .
\end{aligned}
$$


Substituting in (3.4) one hence finds for $\mathscr{W}(\beta)$

$$
\begin{aligned}
\mathscr{W}(\beta)= & \frac{1}{2} \alpha\left\{\cos ^{2} \varphi \sum_{j=1}^{N-1}\left(1-2 c_{j}^{\dagger} c_{j}\right)\right. \\
& +\sin ^{2} \varphi \sum_{j=1}^{N-4}\left(c_{j}^{\dagger}+c_{j}\right)\left(c_{j+4}^{\dagger}-c_{j+4}\right) \\
& -N+\gamma \sum_{j=1}^{N-2}\left(c_{j}^{\dagger}+c_{j}\right)\left(c_{j+2}^{\dagger}-c_{j+2}\right) \\
& +\cos ^{2} \varphi(-1)^{\mathscr{H}_{c}}\left(1-2 c_{N}^{\dagger} c_{N}\right) \\
& +\sin ^{2} \varphi\left[(-1)^{\mathscr{H}_{c}} \sum_{k=1}^{3}\left(c_{N+k-4}^{\dagger}+c_{N+k-4}\right)\left(c_{k}^{\dagger}-c_{k}\right)\right. \\
& \left.+\left(c_{N}^{\dagger}+c_{N}\right)\left(c_{4}^{\dagger}-c_{4}\right)\right] \\
& \left.+\gamma\left[(-1)^{\mathscr{c}_{c}}\left(c_{N-1}^{\dagger}+c_{N-1}\right)\left(c_{1}^{\dagger}-c_{1}\right)+\left(c_{N}^{\dagger}+c_{N}\right)\left(c_{2}^{\dagger}-c_{2}\right)\right]\right\} .
\end{aligned}
$$

Since the fermion operators occur in pairs the operator $(-1)^{\mathscr{S}_{c}}$ commutes with $\mathscr{W}(\beta)$ and the eigenstates of $\mathscr{W}(\beta)$ may be divided into two classes, one for which $\mathscr{N}_{c}$ is even and one for which $\mathscr{N}_{c}$ is odd. We define

$$
c_{N+k}=c_{k} \quad(k=1,2,3) .
$$

Then in the subspace with even $c$ parity $\mathfrak{\mathfrak { E }}_{N}^{+}$the eigenstates of $\mathscr{W}(\beta)$ coincide with the eigenstates of the operator $\mathscr{W}^{+}(\beta)$ given by

$$
\begin{aligned}
\mathscr{W}^{+}(\beta)= & \frac{1}{2} \alpha \sum_{j=1}^{N}\left[\cos ^{2} \varphi\left(1-2 c_{j}^{\dagger} c_{j}\right)\right. \\
& \left.+\sin ^{2} \varphi\left(c_{j}^{\dagger}+c_{j}\right)\left(c_{j+4}^{\dagger}-c_{j+4}\right)-1+\gamma\left(c_{j}^{\dagger}+c_{j}\right)\left(c_{j+2}^{\dagger}-c_{j+2}\right)\right] .
\end{aligned}
$$

The eigenstates of $\mathscr{W}^{+}(\beta)$ with odd $c$ parity are irrelevant and must be discarded. We define new fermion operators $d_{j}^{\dagger}$ and $d_{j}$ by

$$
\begin{aligned}
& d_{j}=c_{j} \quad(j=1, \ldots, N-1), \\
& d_{N}=-c_{N}^{\dagger}, \\
& d_{N+k}=-d_{k} \quad(k=1,2,3) .
\end{aligned}
$$


Then in the subspace with odd $c$ parity $\mathfrak{G}_{N}^{-}$the eigenstates of $\mathscr{W}(\beta)$ coincide with the eigenstates of the operator $\mathscr{W}^{-}(\beta)$ obtained from the expression for $\mathscr{W}^{+}(\beta)$ by replacing the $\left(c^{\dagger}, c\right)$ operators by $\left(d^{\dagger}, d\right)$ operators. The eigenstates of $\mathscr{W}^{-}(\beta)$ with even $c$ parity are irrelevant.

The translational symmetry of $\mathscr{W}^{ \pm}(\beta)$ suggests a transformation to wave operators

$$
\begin{aligned}
& c_{j}=N^{-\frac{1}{2}} \sum_{q} \mathrm{e}^{\mathrm{j} q j} \eta_{q}, \\
& d_{j}=N^{-\frac{1}{2}} \sum_{q} \mathrm{e}^{\mathrm{i} q J} \eta_{q},
\end{aligned}
$$

where in (3.24a) the cyclic condition (3.21) requires $q$ values

$$
q=0, \pm 2 \pi / N, \quad \pm 4 \pi / N, \ldots, \pm(N-2) \pi / N, \pi,
$$

while in (3.24b) the anticyclic condition (3.23) requires

$$
q= \pm \pi / N, \quad \pm 3 \pi / N, \ldots, \pm(N-1) \pi / N .
$$

Substitution yields

$$
\mathscr{W}^{ \pm}(\beta)=-\alpha \sum_{0 \leqslant q \leqslant \pi} \mathscr{W}_{q}(\beta),
$$

where for $\mathscr{W}^{+}(\beta)$ the sum runs over the non-negative $q$ values (3.25a) and for $\mathscr{W}^{-}(\beta)$ the sum runs over the positive $q$ values (3.25b). For $q \neq 0$ or $\pi$

$$
\mathscr{W}_{q}(\beta)=C_{q}\left(\eta_{q}^{\dagger} \eta_{q}+\eta_{-q}^{\dagger} \eta_{-q}-1\right)-\mathrm{i} D_{q}\left(\eta_{q}^{\dagger} \eta_{-q}^{\dagger}+\eta_{q} \eta_{-q}\right)+1,
$$

with

$$
\begin{aligned}
& C_{q}=\cos ^{2} \varphi+\sin ^{2} \varphi \cos 4 q+\gamma \cos 2 q, \\
& D_{q}=\sin ^{2} \varphi \sin 4 q+\gamma \sin 2 q .
\end{aligned}
$$

For $q=0$ or $\pi$

$$
\begin{aligned}
& \mathscr{W}_{0}(\beta)=\frac{1}{2}\left[C_{0}\left(2 \eta_{0}^{\dagger} \eta_{0}-1\right)+1\right], \\
& \mathscr{W}_{\pi}(\beta)=\frac{1}{2}\left[C_{\pi}\left(2 \eta_{\pi}^{\dagger} \eta_{\pi}-1\right)+1\right] .
\end{aligned}
$$

Complete diagonalization is achieved by a Bogoliubov-Valatin transformation

$$
\begin{aligned}
& \xi_{q}=u_{q} \eta_{q}+\mathrm{i} v_{q} \eta_{-q}^{\dagger}, \\
& \xi_{-q}^{\dagger}=\mathrm{i} v_{q} \eta_{q}+u_{q} \eta_{-q}^{\dagger},
\end{aligned}
$$


where $u_{q}=\cos \frac{1}{2} \chi_{q}$ and $v_{q}=-\sin \frac{1}{2} \chi_{q}$ with

$$
\begin{aligned}
& \cos \chi_{q}=C_{q} /\left(C_{q}^{2}+D_{q}^{2}\right)^{\frac{1}{2}}, \\
& \sin \chi_{q}=D_{q} /\left(C_{q}^{2}+D_{q}^{2}\right)^{\frac{1}{2}}, \\
& \left(C_{q}^{2}+D_{q}^{2}\right)^{\frac{1}{2}}=1+\gamma \cos 2 q .
\end{aligned}
$$

In terms of $\xi$ operators $\mathscr{W}_{q}(\beta)$ takes the form $(q \neq 0$ or $\pi)$

$$
\mathscr{W}_{q}(\beta)=-(1+\gamma \cos 2 q)\left(\xi_{q}^{\dagger} \xi_{q}+\xi_{-q}^{\dagger} \xi_{-q}-1\right)-1 .
$$

For $q=0$ or $\pi$ one has $\xi_{0}=\eta_{0}$ and $\xi_{\pi}=\eta_{\pi}$ so that $\mathscr{W}_{0}$ and $\mathscr{W}_{\pi}$ keep the simple form given in (3.29). Finally we may write

$$
\mathscr{W}^{ \pm}(\beta)=-\alpha \sum_{-\pi<q \leqslant \pi}(1+\gamma \cos 2 q) \xi_{q}^{\dagger} \xi_{q},
$$

where for $\mathscr{W}^{+}(\beta)$ the sum runs over the $q$ values (3.25a) and for $\mathscr{W}^{-}(\beta)$ over the $q$ values $(3.25 \mathrm{~b})$.

From (3.10) we know that the states $\left|0_{ \pm}\right\rangle$are eigenstates of $\mathscr{W}(\beta)$ with eigenvalue zero. We now show that $\left|0_{+}\right\rangle$and $\left|0_{-}\right\rangle$lie in $\mathfrak{S}_{N}^{-}$and $\mathfrak{S}_{N}^{+}$, respectively, and are therefore eigenstates with eigenvalue zero of $\mathscr{W}^{-}(\beta)$ and $\mathscr{W}^{+}(\beta)$, respectively. From (3.18) it follows that $\left|1_{-}\right\rangle$has even $c$ parity and $\left|1_{+}\right\rangle$has odd $c$ parity, and hence even $d$ parity. From (3.13) and (3.14) one concludes that the energy operator (3.2) contains an even number of $c$ operators so that $c$ parity and $d$ parity are conserved in the similarity transformation (3.7). This proves our statement and hence

$$
\mathscr{W}^{ \pm}(\beta)\left|0_{\mp}\right\rangle=0 .
$$

For $q$ from the set (3.25a) (for short, $q$ even) $\xi$ parity coincides with $c$ parity while for $q$ from the set (3.25b) (for short, $q$ odd) $\xi$ parity coincides with $d$ parity. From (3.33) and (3.34) it follows that

$$
\xi_{q}\left|0_{ \pm}\right\rangle=0 \quad q \text { even. }
$$

Hence the eigenstates of $\mathscr{W}(\beta)$ are given by

$$
\left|\boldsymbol{q}_{2 k}\right\rangle=\xi_{q_{1}}^{\dagger} \xi_{a_{2}}^{\dagger} \cdots \xi_{q_{2 k}}^{\dagger}\left|0_{\mp}\right\rangle,
$$

where for $\left|0_{-}\right\rangle$all $q$ values are even and for $\left|0_{+}\right\rangle$all $q$ values are odd. As in I. the notation $\boldsymbol{q}_{m}$ is shorthand for an ordered set of $q$ values $\left(q_{1}<q_{2}<\cdots<q_{m}\right)$. We may finally write

$$
\mathscr{W}(\beta)\left|\boldsymbol{q}_{2 k}\right\rangle=-\Lambda\left(\boldsymbol{q}_{2 k}\right)\left|\boldsymbol{q}_{2 k}\right\rangle,
$$


with

$$
\begin{aligned}
& \Lambda\left(\boldsymbol{q}_{2 k}\right)=\sum_{i=1}^{2 k} \lambda_{\boldsymbol{q}_{i}}, \\
& \lambda_{q}=\alpha(1+\gamma \cos 2 q) .
\end{aligned}
$$

In principle we have found the explicit solution of the master equation. Nevertheless our task is not completed since according to the scheme developed so far the time dependence of averages of spin operators can be found only after rather complicated transformations. It will be shown that, as in the case of the 1-flip model, the amount of algebra can be reduced considerably and a simple algorithm can be constructed.

4. Hierarchies of spin operators. An arbitrary operator $A^{z}$ diagonal in the $\sigma^{2}$ representation may be written

$$
A^{z}=\sum_{k, n_{k}} a\left(n_{k}\right) \sigma^{z}\left(n_{k}\right)
$$

where

$$
\sigma^{z}\left(n_{k}\right)=\sigma_{n_{1}}^{z} \sigma_{n_{2}}^{z} \cdots \sigma_{n_{k}}^{z}
$$

and where the sum runs over all ordered sets of integers $n_{k} \equiv\left(n_{1}, n_{2}, \ldots, n_{k}\right)$ with $\left(1 \leqslant n_{1}<n_{2}<\cdots<n_{k} \leqslant N\right)$. A set of integers which is not necessarily ordered will be denoted by $N_{k}$. The average of the observable $A^{z}$ over the probability distribution $P\left(s_{N}, t\right)$ may be written

$$
\left\langle A^{z}\right\rangle_{t}=\sum_{s_{N}} \sum_{k, n_{k}} a\left(n_{k}\right) s\left(n_{k}\right) P\left(s_{N}, t\right)=\left\langle 1\left|A^{z}\right| P(t)\right\rangle,
$$

which may be transformed to

$$
\begin{aligned}
\left\langle A^{z}\right\rangle_{t} & =\left\langle 1_{+}\left|A^{z}\right| P(t)\right\rangle+\left\langle 1_{-}\left|A^{z}\right| P(t)\right\rangle \\
& =\left(Z_{N+} \mid Z_{N}\right)\left\langle 0_{+}\left|A^{z}\right| \psi(t)\right\rangle+\left(Z_{N-} \mid Z_{N}\right)\left\langle 0_{-}\left|A^{z}\right| \psi(t)\right\rangle .
\end{aligned}
$$

From the preceding section we know that

$$
|\psi(t)\rangle=\sum_{k, q_{2 k}} p\left(\boldsymbol{q}_{2 k}\right) \mathrm{e}^{-\lambda\left(\boldsymbol{q}_{2 k}\right) t}\left|\boldsymbol{q}_{2 k}\right\rangle
$$

where the sum is over both the set of even and the set of odd $q$ values and the expansion coefficients $p\left(\boldsymbol{q}_{2 k}\right)$ may be found from the probability distribution at time $t=0$,

$$
p\left(\boldsymbol{q}_{2 k}\right)=\left\langle\boldsymbol{q}_{2 k} \mid \psi(0)\right\rangle=\left\langle\boldsymbol{q}_{2 k}\left|\varrho_{\mathrm{eq}}^{-\frac{1}{2}}\right| P(0)\right\rangle .
$$


Therefore our task is to find simple expressions for the matrix elements $\left\langle 0_{ \pm}\left|\boldsymbol{A}^{\boldsymbol{z}}\right| \boldsymbol{q}_{2 k}\right\rangle$.

We proceed in the same manner as in I and show that the excited states $\left|\boldsymbol{q}_{k}\right\rangle$ may also be obtained from the two vacuum states $\left|0_{ \pm}\right\rangle$by acting on these states with certain products of $\sigma^{z}$ operators. First we relate the excited states to the two unit states. The energy operator $\mathscr{H}_{N}$ may be written

$$
\begin{aligned}
\mathscr{H}_{N} & =\mathscr{H}_{N}^{ \pm}-J\left[(-1)^{\mathscr{N}_{c}} \mp 1\right]\left(c_{N-1}^{\dagger}+c_{N-1}\right)\left(c_{1}^{\dagger}-c_{1}\right) \\
& =\mathscr{H}_{N}^{+}-J\left(1 \pm \prod_{j=1}^{N} \sigma_{j}^{y}\right) \sigma_{N}^{z} \sigma_{1}^{z},
\end{aligned}
$$

where $\mathscr{H}_{N}^{+}$is cyclic in terms of $c$ operators and $\mathscr{H}_{N}^{-}$is cyclic in terms of $d$ operators. $\mathscr{H}_{N}$ coincides with $\mathscr{H}_{N}^{+}$in $\mathfrak{S}_{N}^{+}$and with $\mathscr{H}_{N}^{-}$in $\mathfrak{S}_{N}^{-}$. We define corresponding density operators

$$
\varrho_{\mathrm{eq}}^{ \pm}=\exp \left[-\beta \mathscr{H}_{\mathrm{N}}^{ \pm}\right] / Z_{N}^{ \pm} .
$$

One may also write

$$
\begin{aligned}
\exp \left[-\beta \mathscr{H}_{N}^{ \pm}\right]= & \left\{\frac{1}{2}\left(1 \mp \Pi^{y}\right)+\frac{1}{2}\left(1 \pm \Pi^{y}\right)\left[\cosh 2 K-\sigma_{N}^{z} \sigma_{1}^{z} \sinh 2 K\right]\right\} \\
& \times \exp \left[-\beta \mathscr{H}_{N}\right] .
\end{aligned}
$$

Hence the partition functions $Z_{N}^{ \pm}$are easily evaluated by the transfer-matrix method and one finds

$$
Z_{N}^{ \pm}=\operatorname{Tr} \exp \left[-\beta \mathscr{H}_{N}^{ \pm}\right]=Z_{N} /\left(1+a^{N}\right) .
$$

Using $\Pi^{y}\left|1_{ \pm}\right\rangle= \pm\left|1_{ \pm}\right\rangle$one may write the vacuum states $\left|0_{ \pm}\right\rangle$

$$
\left|0_{ \pm}\right\rangle=\left(Z_{N} Z_{N}^{\mp} / Z_{N_{ \pm}}^{2}\right)^{\frac{1}{2}}\left(\varrho_{\mathrm{eq}}^{\mp}\right)^{\frac{1}{2}}\left|1_{ \pm}\right\rangle
$$

and the excited states $\left|\boldsymbol{q}_{k}\right\rangle$

$$
\left|\boldsymbol{q}_{k}\right\rangle=\xi^{\dagger}\left(\boldsymbol{q}_{k}\right)\left|0_{ \pm}\right\rangle=\left(Z_{N} Z_{N}^{\mp} / Z_{N \pm}^{2}\right)^{\frac{1}{2}}\left(\varrho_{\mathrm{eq}}^{\mp}\right)^{\frac{1}{2}} \xi^{\dagger}\left(\boldsymbol{q}_{k}, \beta\right)\left|1_{ \pm}\right\rangle,
$$

where for the upper (lower) sign a set of odd (even) $q$ values is used and where

$$
\begin{aligned}
& \xi^{\dagger}\left(\boldsymbol{q}_{k}\right)=\xi_{q_{1}}^{\dagger} \xi_{q_{2}}^{\dagger} \cdots \xi_{q_{k}}^{\dagger}, \\
& \xi^{\dagger}\left(\boldsymbol{q}_{k}, \beta\right)=\left(\varrho_{\mathrm{eq}}^{\mp}\right)^{-\frac{1}{2}} \xi^{\dagger}\left(\boldsymbol{q}_{k}\right)\left(\varrho_{\mathrm{eq}}^{\mp}\right)^{\frac{1}{2}} .
\end{aligned}
$$

The operators $\xi^{\dagger}\left(\boldsymbol{q}_{k}, \beta\right)$ are easily expressed in terms of $\eta$ operators by rewriting the energy operators $\mathscr{H}_{N}^{ \pm}$in terms of $\xi$ operators. One finds

$$
\mathscr{H}_{N}^{ \pm}=\sum_{0 \leqslant q \leqslant \pi} \mathscr{H}_{q} \text {, }
$$


where for the upper (lower) sign the sum runs over even (odd) $q$ values and where for $q \neq 0$ or $\pi$

$$
\mathscr{H}_{q}=2 J\left[\cos \psi_{q}\left(\xi_{q}^{\dagger} \xi_{q}+\xi_{-q}^{\dagger} \xi_{-q}-1\right)+i \sin \psi_{q}\left(\xi_{q}^{\dagger} \xi_{-q}^{\dagger}+\xi_{q} \xi_{-q}\right)\right],
$$

with

$$
\psi_{q}=\chi_{q}-2 q,
$$

while for $q=0$ or $\pi$

$$
\begin{aligned}
& \mathscr{H}_{0}=J\left[2 \xi_{0}^{\dagger} \xi_{0}-1\right], \\
& \mathscr{H}_{\pi}=J\left[2 \xi_{\pi}^{\dagger} \xi_{\pi}-1\right] .
\end{aligned}
$$

Hence one finds by straightforward calculation in the same manner as in I

$$
\begin{aligned}
& \xi_{q}^{\dagger}(\beta)=g_{q}^{-1}\left[\eta_{q}^{\dagger}+\mathrm{i} f_{q} \eta_{-q}\right], \\
& \xi_{q}(\beta)=g_{q} \eta_{q},
\end{aligned}
$$

where

$$
\begin{aligned}
& g_{q}=(\cosh 2 K)^{-\frac{1}{2}}(1+\gamma \cos 2 q)^{-\frac{1}{2}}, \\
& f_{q}=\gamma \sin 2 q /(1+\gamma \cos 2 q) .
\end{aligned}
$$

Note that (4.18b) proves the consistency of (3.18) and (3.35).

The relation (4.18a) will be used in (4.12). The effect of the creation operators $\eta_{a}^{\dagger}$ on the unit states is expressed most conveniently with the aid of a $\sigma \tau$ transformation $^{6}$ ) of order 2 . The $\sigma$ and $\tau$ operators in this transformation are related by

$$
\begin{aligned}
\tau_{j}^{x} & =\sigma_{j}^{x} \sigma_{j+1}^{x}, \\
\tau_{j}^{y} & =\sigma_{1}^{z} \sigma_{2}^{z} \cdots \sigma_{J-1}^{z} \sigma_{J}^{y} \sigma_{j+1}^{x}, \\
\tau_{j}^{z} & =\sigma_{1}^{z} \sigma_{2}^{z} \cdots \sigma_{J}^{z} \quad(j=1,2, \ldots, N), \quad \sigma_{N+1}^{x} \equiv 1 .
\end{aligned}
$$

The inverse transformation reads

$$
\begin{aligned}
& \sigma_{j}^{x}=\tau_{j}^{x} \tau_{j+1}^{x} \cdots \tau_{N}^{x}, \\
& \sigma_{j}^{y}=\tau_{j-1}^{z} \tau_{j}^{y} \tau_{j+1}^{x} \tau_{j+2}^{x} \cdots \tau_{N}^{x}, \\
& \sigma_{j}^{z}=\tau_{j-1}^{z} \tau_{j}^{z} \quad(j=1, \ldots, N), \quad \tau_{0}^{z} \equiv 1 .
\end{aligned}
$$

From (2.18) and (3.17) one easily finds

$$
\begin{aligned}
& c_{2 l-1}^{\dagger}\left|1_{ \pm}\right\rangle=d_{2 l-1}^{\dagger}\left|1_{ \pm}\right\rangle=\mathrm{i}^{-(2 l-1)} \tau_{2 l-1}^{z}\left|1_{ \pm}\right\rangle, \\
& c_{2 l}^{\dagger}\left|1_{ \pm}\right\rangle=d_{2 l}^{\dagger}\left|1_{ \pm}\right\rangle=\mathrm{i}^{-2 l} \tau_{2 l}^{z}\left|1_{\mp}\right\rangle \quad(2 l=2,4, \ldots, N-2), \\
& c_{N}^{\dagger}\left|1_{-}\right\rangle=\left|1_{+}\right\rangle, \quad d_{N}^{\dagger}\left|1_{+}\right\rangle=-\left|1_{-}\right\rangle .
\end{aligned}
$$


Similarly the successive action of a number of $c^{\dagger}$ or $d^{\dagger}$ operators on the unit statcs may be replaced by the action of a product of $\tau^{z}$ operators. We define

$$
\begin{aligned}
& \hat{\tau}^{z}\left(n_{2 l}\right)=(-1)^{l} \mathrm{i}^{v\left(n_{2 l}\right)} \tau^{z}\left(n_{2 l}\right), \\
& \hat{\tau}^{z}\left(n_{2 l+1}\right)=(-1)^{l} \mathrm{i}^{-v\left(n_{2 l+1}\right)} \tau^{z}\left(n_{2 l+1}\right),
\end{aligned}
$$

where $\tau^{z}\left(\boldsymbol{n}_{k}\right)$ is defined in the same manner as $\sigma^{z}\left(\boldsymbol{n}_{k}\right)$ in (4.2) and where the exponent $v\left(n_{k}\right)$ is defined by

$$
\begin{aligned}
& \nu\left(n_{2 l}\right)=\sum_{j=1}^{l}\left(n_{2 j-1}-n_{2 j}\right), \\
& \nu\left(n_{2 l+1}\right)=\sum_{j=1}^{l}\left(n_{2 j-1}-n_{2 j}\right)+n_{2 l+1} .
\end{aligned}
$$

We write the operator $\hat{\tau}^{z}\left(n_{k}\right)$ as a sum of two parts

$$
\begin{gathered}
\hat{\tau}^{z}\left(n_{k}\right)=\hat{\tau}_{1}^{z}\left(n_{k}\right)+\hat{\tau}_{2}^{z}\left(n_{k}\right), \\
\begin{array}{ccc}
\hat{\tau}_{1,2}^{z}\left(n_{2 l}\right)=\hat{\tau}^{z}\left(n_{2 l}\right) & \text { for } & \sum_{j=1}^{2 l} n_{j} \quad \text { even (odd), } \\
=0 & \text { for } & \sum_{j=1}^{2 l} n_{j} \quad \text { odd (even), } \\
\hat{\tau}_{1,2}^{z}\left(n_{2 l+1}\right)=\hat{t}^{z}\left(n_{2 l+1}\right) & \text { for } \sum_{j=1}^{2 l+1} n_{j} \quad \text { odd (even), } \\
=0 & \text { for } \sum_{j=1}^{2 l+1} n_{j} \quad \text { even (odd). }
\end{array}
\end{gathered}
$$

With these conventions the action of an ordered product of $c^{\dagger}$ or $d^{\dagger}$ operators on the unit states is expressed by

$$
\begin{aligned}
& c^{\dagger}\left(n_{k}\right)\left|1_{-}\right\rangle=\hat{\tau}_{1}^{z}\left(n_{k}\right)\left|1_{-}\right\rangle+\hat{\tau}_{2}^{z}\left(n_{k}\right)\left|1_{+}\right\rangle, \\
& d^{\dagger}\left(n_{k}\right)\left|1_{+}\right\rangle=\hat{\tau}_{1}^{z}\left(n_{k}\right)\left|1_{+}\right\rangle+\hat{\tau}_{2}^{z}\left(n_{k}\right)\left|1_{-}\right\rangle .
\end{aligned}
$$

For a not necessarily ordered sequence of integers $N_{k} \equiv\left(N_{1}, N_{2}, \ldots, N_{k}\right)$ we define

$$
\hat{\tau}_{1,2}^{z}\left(N_{k}\right)=(-1)^{P\left(N_{k}\right)} \hat{\tau}_{1,2}^{z}\left(n_{k}\right),
$$

where $(-1)^{P\left(N_{k}\right)}$ is the sign of the permutation needed to bring $N_{k}$ to normal order $\boldsymbol{n}_{k}$. Then in terms of Fourier transforms (4.26) may be written

$$
\eta^{\dagger}\left(q_{k}\right)\left|1_{ \pm}\right\rangle=T_{1}^{z}\left(q_{k}, 0\right)\left|1_{ \pm}\right\rangle+T_{2}^{z}\left(q_{k}, 0\right)\left|1_{\mp}\right\rangle, \quad q_{\text {cren }}^{\text {odd }} .
$$


with

$$
T_{1,2}^{\mathrm{z}}\left(\boldsymbol{q}_{k}, 0\right)=N^{-\frac{1}{2} k} \sum_{\boldsymbol{n}_{k}} D\left(\boldsymbol{q}_{k}, \boldsymbol{n}_{k}\right) \hat{\tau}_{1,2}^{\mathrm{z}}\left(\boldsymbol{n}_{k}\right),
$$

where the sum is over all ordered $k$-tuples $\boldsymbol{n}_{k}$ and where $\boldsymbol{D}\left(\boldsymbol{q}_{k}, \boldsymbol{n}_{k}\right)$ is the $(k \times k)$ Slater determinant

$$
D\left(q_{k}, n_{k}\right)=\operatorname{det}_{k}\left(\mathrm{e}^{\mathrm{i} q_{j} n_{l}}\right),
$$

with rows in normal order $\boldsymbol{q}_{k}$ and columns in normal order $\boldsymbol{n}_{k}$. It will be shown later that $T_{1,2}^{z}\left(q_{k}, 0\right)$ can naturally be regarded as the $\beta=0$ limit of a more general operator $T_{1,2}^{z}\left(\boldsymbol{q}_{k}, \beta\right)$. From the definitions (4.25) and (4.29) it follows that

$$
T_{1,2}^{z}\left(q_{k}+\pi, 0\right)= \pm(-1)^{k} T_{1,2}^{z}\left(q_{k}, 0\right),
$$

where the wave numbers $q_{j}+\pi$ are defined modulo $(2 \pi)$. In combination with (4.28) this yields

$$
\begin{array}{ll}
T_{1}^{z}\left(\boldsymbol{q}_{k}, 0\right)\left|1_{ \pm}\right\rangle=\frac{1}{2}\left[\eta^{\dagger}\left(\boldsymbol{q}_{k}\right)+(-1)^{k} \eta^{\dagger}\left(\boldsymbol{q}_{k}+\pi\right)\right]\left|1_{ \pm}\right\rangle & q_{\substack{\text { oven } \\
\text { even }}}^{\text {, }}, \\
T_{2}^{z}\left(\boldsymbol{q}_{k}, 0\right)\left|1_{ \pm}\right\rangle=\frac{1}{2}\left[\eta^{\dagger}\left(\boldsymbol{q}_{k}\right)-(-1)^{k} \eta^{\dagger}\left(\boldsymbol{q}_{k}+\pi\right)\right]\left|1_{\mp}\right\rangle & q_{\text {odd }}^{\text {even }} .
\end{array}
$$

Since $\Pi^{z}$ commutes with the $T^{z}$ operators these states are eigenstates of the $\Pi^{z}$ operator. From (4.28) and (4.31) one finds using (3.18) and the anticommutation relations for the $\eta$ operators that the operators $T_{1,2}^{z}\left(q_{k}, 0\right)$ satisfy the orthonormality relations

$$
\begin{aligned}
& \left\langle 1_{ \pm}\left|T_{1}^{z}\left(\boldsymbol{q}_{k}, 0\right)^{\dagger} T_{1}^{z}\left(\boldsymbol{q}_{l}^{\prime}, 0\right)\right| 1_{ \pm}\right\rangle \\
& =2^{N-2} \delta_{k l}\left[\delta\left(\boldsymbol{q}_{k}-\boldsymbol{q}_{k}^{\prime}\right)+(-1)^{k} \delta\left(\boldsymbol{q}_{k}+\pi-\boldsymbol{q}_{k}^{\prime}\right)\right] \quad \boldsymbol{q}_{\text {even }}^{\text {odd }}, \\
& \left\langle 1_{ \pm}\left|T_{2}^{z}\left(q_{k}, 0\right)^{\dagger} T_{2}^{z}\left(q_{1}^{\prime}, 0\right)\right| 1_{ \pm}\right\rangle \\
& =2^{N-2} \delta_{k l}\left[\delta\left(\boldsymbol{q}_{k}-\boldsymbol{q}_{k}^{\prime}\right)-(-1)^{k} \delta\left(\boldsymbol{q}_{k}+\boldsymbol{\pi}-\boldsymbol{q}_{k}^{\prime}\right)\right] \quad \boldsymbol{q}_{\text {odd }}^{\text {even }} .
\end{aligned}
$$

It is easily checked that all matrix elements of the operators $\hat{\tau}_{1}^{z}\left(n_{k}\right)$ and $\hat{\tau}_{2}^{z}\left(n_{k}\right)$ between the states $\left|1_{+}\right\rangle$and $\left|1_{-}\right\rangle$vanish except for $\hat{\tau}_{2}^{z}(N)=\tau_{N}^{z}=\Pi^{z}$ for which

$$
\left\langle 1_{ \pm}\left|\tau_{N}^{z}\right| 1_{ \pm}\right\rangle= \pm 2^{N-1} .
$$

Hence we have

$$
\begin{aligned}
& \left\langle 1_{ \pm}\left|T_{1}^{z}\left(\boldsymbol{q}_{k}, 0\right)\right| 1_{ \pm}\right\rangle=0 \quad(k=1,2, \ldots), \\
& \left\langle 1_{\mp}\left|T_{2}^{z}\left(\boldsymbol{q}_{k}, 0\right)\right| 1_{\mp}\right\rangle=0 \quad(k=2,3, \ldots), \\
& \left\langle 1_{\mp}\left|T_{2}^{z}(q, 0)\right| 1_{\mp}\right\rangle=2^{N-1} N^{-\frac{1}{2}},
\end{aligned}
$$


where in all three relations the $q$ 's. are odd for the upper sign and even for the lower sign. We define in addition for $k=0$

$$
T_{1}^{z}\left(q_{0}, 0\right)=T_{2}^{z}\left(q_{0}, 0\right)=1,
$$

but note that the relations (4.33) must be modified for $k$ or $l=0$ to take account of the relations (4.35).

We relate the members of the hierarchies of operators $T_{1}^{2}\left(q_{k}, 0\right)$ and $T_{2}^{z}\left(q_{k}, 0\right)$ for different values of $k$ by means of creation and annihilation superoperators defined by

$$
\Gamma_{\alpha, q}^{\dagger} T_{\alpha}^{z}\left(q_{k}, 0\right)=(-1)^{j-1} T_{\alpha}^{z}\left(q_{k+1}, 0\right),
$$

where

$$
\begin{aligned}
\boldsymbol{q}_{k+1} & \equiv\left(q_{1}<\cdots<q_{j-1}<q<q_{j}<\cdots<q_{k}\right) \\
& =0 \text { if } \quad q \in \boldsymbol{q}_{k}, \\
\Gamma_{\alpha, q} T_{\alpha}^{z}\left(\boldsymbol{q}_{k}, 0\right) & =(-1)^{j-1} T_{\alpha}^{z}\left(\boldsymbol{q}_{k-1}, 0\right),
\end{aligned}
$$

where

$$
\begin{aligned}
\boldsymbol{q}_{k-1} & \equiv\left(q_{1}<\cdots<q_{j-1}<q_{j+1}<\cdots<q_{k}\right) \text { for } q=q_{j} \\
& =0 \quad \text { if } \quad q \notin q_{k} \quad(\alpha=1,2) .
\end{aligned}
$$

It is easily verified that the operators $\Gamma_{\alpha, q}^{\dagger}$ and $\Gamma_{\alpha, q}$ satisfy the Fermi anticommutation rules.

We return to the consideration of the state $\xi^{\dagger}\left(q_{k}, \beta\right)\left|1_{ \pm}\right\rangle$in (4.12). Using (4.18a), bringing the products of $\eta^{\dagger}$ and $\eta$ operators to normal order and applying (4.28), it follows in the same manner as in I that one may write

$$
\xi^{\dagger}\left(q_{k}, \beta\right)\left|1_{ \pm}\right\rangle=T_{1}^{z}\left(q_{k}, \beta\right)\left|1_{ \pm}\right\rangle+T_{2}^{z}\left(q_{k}, \beta\right)\left|1_{\mp}\right\rangle \quad q_{\text {even }}^{\text {odd }},
$$

where the operators $T_{1,2}^{z}\left(q_{k}, \beta\right)$ are related to the $T_{1,2}^{z}\left(q_{k}, 0\right)$ by

$$
T_{\alpha}^{z}\left(\boldsymbol{q}_{k}, \beta\right)=g\left(\boldsymbol{q}_{k}\right)^{-1} \Omega_{\alpha} T_{\alpha}^{z}\left(\boldsymbol{q}_{k}, 0\right) \quad(\alpha=1,2),
$$

with

$$
g\left(q_{k}\right)=\prod_{j=1}^{k} g\left(q_{j}\right)
$$

and the superoperator $\Omega_{\alpha}$ given by

$$
\Omega_{\alpha}=\exp \left[-\mathrm{i} \sum_{0<q<\pi} f_{q} \Gamma_{\alpha, q} \Gamma_{\alpha,-q}\right] \quad(\alpha=1,2) .
$$


The explicit expressions for the first few members of the hierarchies read

$$
\begin{aligned}
& T_{\alpha}^{z}\left(q_{0}, \beta\right)=T_{\alpha}^{z}\left(q_{0}, 0\right)=1, \\
& T_{\alpha}^{z}\left(q_{1}, \beta\right)=g\left(q_{1}\right)^{-1} T_{\alpha}^{z}\left(q_{1}, 0\right), \\
& T_{\alpha}^{z}\left(q_{2} ; \beta\right)=g\left(q_{2}\right)^{-1}\left[T_{\alpha}^{z}\left(q_{2}, 0\right)+\mathrm{i} f_{q_{1}} \delta\left(q_{1}+q_{2}\right)\right] .
\end{aligned}
$$

Higher members of the hierarchies may be found by successive application of a superoperator, as in (I.8.12) and (I.8.14). We refer to I for the explicit expressions for $k=3$ and 4 .

The expression (4.38) may be substituted in (4.12) and we now wish to commute the operators $\left(\varrho_{\mathrm{eq}}^{\mp}\right)^{\frac{1}{2}}$ and $T_{\alpha}^{\mathrm{z}}\left(q_{k}, \beta\right)$. From (4.7) it follows that

$$
\left(\varrho_{\mathrm{eq}}^{ \pm}\right)^{\frac{1}{2}}=\left(Z_{N} / Z_{N}^{ \pm}\right)^{\frac{1}{2}} \varrho_{\mathrm{eq}}^{\frac{1}{2}} C^{ \pm},
$$

where

$$
C^{ \pm}=\frac{1}{2}\left(1 \mp \Pi^{y}\right)+\frac{1}{2}\left(1 \pm \Pi^{y}\right)\left[\cosh K-\sigma_{N}^{z} \sigma_{1}^{z} \sinh K\right] .
$$

The only operator in (4.43) that does not commute with $T_{\alpha}^{z}\left(q_{k}, \beta\right)$ is

$$
\Pi^{y}=\prod_{j=1}^{N} \sigma_{J}^{y}=\tau_{1}^{x} \tau_{3}^{x} \tau_{5}^{x} \cdots \tau_{N-1}^{x} \tau_{N}^{z}
$$

One easily finds from (4.25)

$$
\Pi^{y} \hat{\tau}_{1,2}^{z}\left(n_{k}\right)= \pm(-1)^{k} \hat{\tau}_{1,2}^{z}\left(n_{k}\right) \Pi^{y},
$$

so that

$$
\begin{array}{ll}
C^{ \pm} \hat{\tau}_{1}^{z}\left(\boldsymbol{n}_{2 l}\right)=\hat{\tau}_{1}^{z}\left(\boldsymbol{n}_{2 l}\right) C^{ \pm}, & C^{ \pm} \hat{\tau}_{1}^{z}\left(\boldsymbol{n}_{2 l+1}\right)=\hat{\tau}_{1}^{z}\left(\boldsymbol{n}_{2 l+1}\right) C^{\mp}, \\
C^{ \pm} \hat{\tau}_{2}^{z}\left(\boldsymbol{n}_{2 l}\right)=\hat{\tau}_{2}^{z}\left(\boldsymbol{n}_{2 l}\right) C^{\mp}, & C^{ \pm} \hat{\tau}_{2}^{z}\left(\boldsymbol{n}_{2 l+1}\right)=\hat{\tau}_{2}^{z}\left(\boldsymbol{n}_{2 l+1}\right) C^{ \pm} .
\end{array}
$$

The effect of the operators $C^{ \pm}$on the unit states is given by

$$
\begin{aligned}
& C^{+}\left|1_{+}\right\rangle=\left[\cosh K-\sigma_{N}^{z} \sigma_{1}^{z} \sinh K\right]\left|1_{+}\right\rangle, \\
& C^{-}\left|1_{-}\right\rangle=\left[\cosh K-\sigma_{N}^{z} \sigma_{1}^{z} \sinh K\right]\left|1_{-}\right\rangle, \\
& C^{+}\left|1_{-}\right\rangle=\left|1_{-}\right\rangle, \quad C^{-}\left|1_{+}\right\rangle=\left|1_{+}\right\rangle .
\end{aligned}
$$

Combining these results one finally finds

$$
\left|\boldsymbol{q}_{k}\right\rangle=T_{1}^{z}\left(\boldsymbol{q}_{k}, \beta\right) \varepsilon_{k}^{z}\left|0_{ \pm}\right\rangle+\left(Z_{N_{\mp}} \mid Z_{N_{ \pm}}\right) T_{2}^{z}\left(\boldsymbol{q}_{k}, \beta\right) \varepsilon_{k}^{z}\left|0_{\mp}\right\rangle \quad q_{\text {even }}^{\text {odd }},
$$


where

$$
\begin{aligned}
& \varepsilon_{k}^{z}=1 \quad \text { for } k \text { even, } \\
& \varepsilon_{k}^{z}=\exp \left[-K \sigma_{N}^{z} \sigma_{1}^{z}\right] \quad \text { for } k \text { odd } .
\end{aligned}
$$

Hence one may derive orthonormality relations for the operators $T_{\alpha}^{z}\left(q_{k}, \beta\right)$ analogous to (4.33), namely for $k \geqslant 1, l \geqslant 1$,

$$
\begin{aligned}
& \left\langle 0_{ \pm}\left|\varepsilon_{k}^{z} T_{1}^{z}\left(\boldsymbol{q}_{k}, \beta\right)^{\dagger} T_{1}^{z}\left(\boldsymbol{q}_{l}^{\prime}, \beta\right) \varepsilon_{l}^{z}\right| 0_{ \pm}\right\rangle \\
& =\frac{Z_{N}}{2 Z_{N \pm}} \delta_{k l}\left[\delta\left(\boldsymbol{q}_{k}-\boldsymbol{q}_{k}^{\prime}\right)+(-1)^{k} \delta\left(\boldsymbol{q}_{k}+\pi-\boldsymbol{q}_{k}^{\prime}\right)\right] \quad \boldsymbol{q}_{\text {even }}^{\text {odd }}, \\
& \left\langle 0_{ \pm}\left|\varepsilon_{k}^{z} T_{2}^{z}\left(\boldsymbol{q}_{k}, \beta\right)^{\dagger} T_{2}^{z}\left(\boldsymbol{q}_{l}^{\prime}, \beta\right) \varepsilon_{l}^{z}\right| 0_{ \pm}\right\rangle \\
& \quad=\frac{Z_{N} Z_{N \mp}}{2\left(Z_{N \pm}\right)^{2}} \delta_{k l}\left[\delta\left(\boldsymbol{q}_{k}-\boldsymbol{q}_{k}^{\prime}\right)-(-1)^{k} \delta\left(\boldsymbol{q}_{k}+\pi-\boldsymbol{q}_{k}^{\prime}\right)\right] \quad q_{\text {odd }}^{\text {even }} .
\end{aligned}
$$

For $k$ or $l$ equal to zero these expressions must be modified in analogy to (4.35). After all these preparations we are ready to evaluate the matrix elements $\left\langle 0_{ \pm}\left|A^{z}\right| \boldsymbol{q}_{2 k}\right\rangle$.

5. Time dependence of spin averages. For the calculation of the time dependence of the averages of $A^{z}$ operators we use the expansions derived in the preceding section. First we expand the general operator $A^{z}$ as given by (4.1) in terms of $T^{z}$ operators. We define $A_{ \pm}^{z}$ as the operators obtained from $A^{z}$ by replacing $\sigma_{1}^{z}$ by $\pm \sigma_{2}^{z} \cdots \sigma_{N}^{z}$. From (4.4) it follows that the time-dependent average of the operator $A^{z}$ may be written

$$
\left\langle A^{z}\right\rangle_{t}=\left(Z_{N+} \mid Z_{N}\right)\left\langle 0_{+}\left|A_{+}^{z}\right| \psi(t)\right\rangle+\left(Z_{N-} \mid Z_{N}\right)\left\langle 0_{-}\left|A_{-}^{z}\right| \psi(t)\right\rangle .
$$

Since $\sigma_{1}^{z}$ does not occur in the operators $A_{ \pm}^{z}$ these operators contain sums of products of even numbers of $\tau_{j}^{z}$ operators, as is evident from (4.16). Hence after Fourier transformation $A_{ \pm}^{z}$ may be expanded as

$$
A_{ \pm}^{z}=\sum_{\alpha=1,2} \sum_{k=0}^{\frac{1}{2} N} \sum_{q_{2 k}} d_{ \pm, \alpha}\left(q_{2 k}, 0\right) T_{\alpha}^{z}\left(q_{2 k}, 0\right)
$$

where one may choose to use either a set of even or a set of odd $q$ 's. These expansions may be turned into an expansion in $T_{\alpha}^{z}\left(\boldsymbol{q}_{2 k}, \beta\right)$ operators using the linear 
relationship (4.39) which may be written explicitly

$$
T_{\alpha}^{z}\left(\boldsymbol{q}_{k}, \beta\right)=g\left(\boldsymbol{q}_{k}\right)^{-1} \sum_{i, \boldsymbol{q}_{2}^{\prime}} \omega\left(\boldsymbol{q}_{k}, \boldsymbol{q}_{l}^{\prime}\right) T_{\alpha}^{z}\left(\boldsymbol{q}_{l}^{\prime}, 0\right) .
$$

Inverting this relation and substituting in (5.2) one finds

$$
A_{ \pm}^{z}=\sum_{\alpha=1,2} \sum_{k=0}^{\frac{1}{2} N} \sum_{q_{2 k}} d_{ \pm, \alpha}\left(q_{2 k}, \beta\right) T_{\alpha}^{z}\left(q_{2 k}, \beta\right),
$$

where the expansion coefficients are related to the coefficients in (5.2) by

$$
d_{ \pm, \alpha}\left(\boldsymbol{q}_{2 k}, \beta\right)=g\left(\boldsymbol{q}_{2 k}\right) \sum_{l, \boldsymbol{q}^{\prime} 2 l} \omega^{\dagger}\left(\boldsymbol{q}_{2 k}, \boldsymbol{q}_{2 l}^{\prime}\right) d_{ \pm, \alpha}\left(\boldsymbol{q}_{2 l}^{\prime}, 0\right) .
$$

The matrix elements $\left\langle 0_{ \pm}\left|A_{ \pm}^{z}\right| \boldsymbol{q}_{2 k}\right\rangle$ may now be calculated by using the expansions (5.4) in combination with the expansion (4.49) for the states $\left|\boldsymbol{q}_{2 k}\right\rangle$. First we note that no cross terms between $T_{1}^{z}\left(q_{2 k}, \beta\right)$ operators and $T_{2}^{z}\left(q_{2 l}^{\prime}, \beta\right)$ operators survive. This is a consequence of the relationship

$$
\left\langle 1_{ \pm}\left|\tau_{1}^{z}\left(n_{2 k}\right) \tau_{2}^{z}\left(n_{2 l}^{\prime}\right)\right| 1_{ \pm}\right\rangle=0,
$$

which is easily derived by noting that in terms of $\tau$ spinors the states $\left|1_{ \pm}\right\rangle$read

$$
\begin{aligned}
& \left|1_{+}\right\rangle=\left(\begin{array}{l}
1 \\
1
\end{array}\right)_{1} \times\left(\begin{array}{l}
1 \\
1
\end{array}\right)_{2} \times \cdots \times\left(\begin{array}{l}
1 \\
1
\end{array}\right)_{N-1} \times\left(\begin{array}{l}
1 \\
0
\end{array}\right)_{N} \\
& \left|1_{-}\right\rangle=\left(\begin{array}{l}
1 \\
1
\end{array}\right)_{1} \times\left(\begin{array}{l}
1 \\
1
\end{array}\right)_{2} \times \cdots \times\left(\begin{array}{l}
1 \\
1
\end{array}\right)_{N-1} \times\left(\begin{array}{l}
0 \\
1
\end{array}\right)_{N} .
\end{aligned}
$$

Hence the only operator $\tau^{z}\left(n_{m}\right)$ with nonvanishing expectation value for the states $\left|1_{ \pm}\right\rangle$is $\tau_{N}^{z}$, and this cannot occur in (5.6). Similarly one finds

$$
\begin{aligned}
& \left\langle 0_{ \pm}\left|\tau_{1}^{z}\left(n_{2 k}\right) \tau_{2}^{z}\left(n_{2 l}^{\prime}\right)\right| 0_{ \pm}\right\rangle \\
& \quad=\left(Z_{N} / Z_{N \pm}\right)^{2}\left\langle 1_{ \pm}\left|\varrho_{\mathrm{eq}} \tau_{1}^{z}\left(n_{2 k}\right) \tau_{2}^{z}\left(n_{2 l}^{\prime}\right)\right| 1_{ \pm}\right\rangle=0,
\end{aligned}
$$

since the additional operator $\varrho_{\mathrm{eq}}$ may be written

$$
\varrho_{\mathrm{eq}}=Z_{N}^{-1} \prod_{j=1}^{N-1}\left(\cosh K+\tau_{j-1}^{z} \tau_{j+1}^{z} \sinh K\right)\left(\cosh K+\tau_{N-1}^{z} \tau_{N}^{z} \tau_{1}^{z} \sinh K\right),
$$

where when acting on the states $\left|1_{ \pm}\right\rangle$the operator $\tau_{N}^{z}$ in the last factor may be replaced by \pm 1 , so that $\left\langle 1_{ \pm}\right| \varrho_{\mathrm{eq}} \tau_{1}^{z}\left(n_{2 k}\right)$ is a sum of terms of the form 
$\left\langle 1_{ \pm}\right| \tau_{1}^{z}\left(n_{2 m}\right)$ and we can appeal to (5.6). Therefore we find for the matrix elements $\left\langle 0_{ \pm}\left|A_{ \pm}^{z}\right| q_{2 k}\right\rangle$ using the orthonormality relations (4.51)

$$
\begin{array}{rlr}
\left\langle 0_{+}\left|A_{+}^{z}\right| q_{2 k}\right\rangle= & \left(Z_{N} / 2 Z_{N+}\right) \\
& \times\left[d_{+, 1}\left(-q_{2 k}, \beta\right)+d_{+, 1}\left(-q_{2 k}+\pi, \beta\right)\right] \quad q \text { odd }, \\
\left\langle 0_{+}\left|A_{+}^{z}\right| q_{2 k}\right\rangle= & \left(Z_{N} / 2 Z_{N+}\right) \\
& \times\left[d_{+, 2}\left(-q_{2 k}, \beta\right)-d_{+, 2}\left(-q_{2 k}+\pi, \beta\right)\right] \quad q \text { even, } \\
\left\langle 0_{-}\left|A_{-}^{z}\right| q_{2 k}\right\rangle= & \left(Z_{N} / 2 Z_{N-}\right) & \\
& \times\left[d_{-, 2}\left(-q_{2 k}, \beta\right)-d_{-, 2}\left(-q_{2 k}+\pi, \beta\right)\right] \quad q \text { odd }, \\
\left\langle 0_{-}\left|A_{-}^{z}\right| q_{2 k}\right\rangle= & \left(Z_{N} / 2 Z_{N-}\right) \\
& \times\left[d_{-, 1}\left(-q_{2 k}, \beta\right)+d_{-, 1}\left(-q_{2 k}+\pi, \beta\right)\right] \quad q \text { even. }
\end{array}
$$

Here we have assumed $k>0$. For the equilibrium averages one finds

$$
\begin{aligned}
& \left\langle 0_{+}\left|A_{+}^{z}\right| 0_{+}\right\rangle=\left(Z_{N} / Z_{N+}\right) d_{+}\left(q_{0}, \beta\right), \\
& \left\langle 0_{-}\left|A_{-}^{z}\right| 0_{-}\right\rangle=\left(Z_{N} / Z_{N-}\right) d_{N-}\left(q_{0}, \beta\right),
\end{aligned}
$$

where $d_{ \pm}\left(q_{0}, \beta\right)$ are the constant terms in the expansions (5.4). The complete time dependence of the expectation value $\left\langle A^{z}\right\rangle_{t}$ follows by combining these results with (4.5).

As an example we evaluate the time-dependent average of a Fourier component of the magnetization,

$$
\sigma^{z}(q)=N^{-\frac{1}{2}} \sum_{j=1}^{N} \sigma_{j}^{z} \mathrm{e}^{-1 q j}
$$

Hence we have

$$
\begin{aligned}
\sigma_{ \pm}^{z}(q) & =N^{-\frac{1}{2}}\left[ \pm \mathrm{e}^{-\mathrm{i} q} \sigma_{2}^{z} \cdots \sigma_{N}^{z}+\sum_{j=2}^{N} \sigma_{j}^{z} \mathrm{e}^{-1 q J}\right] \\
& =N^{-\frac{1}{2}}\left[ \pm \mathrm{e}^{-1 q q} \tau_{1}^{z} \tau_{N}^{z}+\sum_{j=2}^{N} \tau_{j-1}^{z} \tau_{j}^{z} \mathrm{e}^{-1 q j}\right] \\
& =N^{-\frac{1}{2}}\left[ \pm \mathrm{i} \mathrm{e}^{-1 q} \hat{\tau}_{2}^{z}(1, N)-\mathrm{i} \sum_{j=2}^{N} \hat{\tau}_{2}^{z}(j-1, j) \mathrm{e}^{-i q j}\right]
\end{aligned}
$$


Choosing $q$ even and using (4.29) one finds

$$
\begin{aligned}
\sigma_{ \pm}^{z}(q)= & -\mathrm{i} N^{-\frac{1}{2}} \sum_{q_{1}<q_{2}} \delta\left(q+q_{1}+q_{2}\right) \\
& \times\left(\mathrm{e}^{1 q_{1}}-\mathrm{e}^{\mathrm{i} q_{2}}\right) T_{2}^{z}\left(q_{1}, q_{2} ; 0\right) \quad\left(q_{1}, q_{2}\right) \text { odd }
\end{aligned}
$$

For $q$ odd the wave numbers $\left(q_{1}, q_{2}\right)$ are odd for $\sigma_{+}^{z}(q)$ and even for $\sigma_{-}^{z}(q)$. From (4.42) and (5.14)

$$
\begin{aligned}
\sigma_{ \pm}^{z}(q)= & -\mathrm{i} N^{-\frac{1}{2}} \sum_{q_{1}<q_{2}} \delta\left(q+q_{1}+q_{2}\right) \\
& \times\left(\mathrm{e}^{\mathrm{i} q_{1}}-\mathrm{e}^{\mathrm{i} q_{2}}\right) g_{q_{1}} g_{q_{2}} T_{2}^{z}\left(q_{1}, q_{2} ; \beta\right) \quad\left(q_{1}, q_{2}\right)_{\text {odd }}^{\text {even }},
\end{aligned}
$$

where we have used

$$
\sum_{q_{1}} f_{q_{1}} \sin q_{1}=0 \quad\left(q_{1} \text { even or odd }\right) .
$$

The only nonvanishing matrix elements (5.10) are therefore given by

$$
\begin{aligned}
& \left\langle 0_{ \pm}\left|\sigma_{ \pm}^{z}(q)\right| q_{1}, q_{2}\right\rangle=-\mathrm{i}\left(Z_{N} / Z_{N \pm}\right) N^{-\frac{1}{2}} \\
& \times \delta\left(q-q_{1}-q_{2}\right)\left(\mathrm{e}^{-\mathrm{i} q_{1}}-\mathrm{e}^{-1 q_{2}}\right) g_{q_{1}} g_{q_{2}} \quad\left(q_{1}, q_{2}\right){ }_{\text {odd }}^{\substack{\text { oven } \\
\text { od }}}
\end{aligned}
$$

Inserting in (5.1) and using (4.5) one finds

$$
\begin{aligned}
\left\langle\sigma^{z}(q)\right\rangle_{t}= & -\mathrm{i} N^{-\frac{1}{2}} \sum_{q_{1}<q_{2}} p\left(q_{1}, q_{2}\right) \delta\left(q-q_{1}-q_{2}\right) \\
& \times\left(\mathrm{e}^{-\mathrm{i} q_{1}}-\mathrm{e}^{-\mathrm{i} q_{2}}\right) g_{q_{1}} g_{q_{2}} \mathrm{e}^{-\Lambda\left(q_{1}, q_{2}\right) t} .
\end{aligned}
$$

The equilibrium time correlation function of two observables $A^{z}$ and $B^{z}$ is defined by

$$
\left\langle A^{z}(t) B^{z}\right\rangle_{\mathrm{eq}, \mu}=\sum_{s_{N^{\prime}}} \sum_{s_{N}} A^{z}\left(s_{N}^{\prime}\right) B^{z}\left(s_{N}\right) P\left(s_{N}^{\prime} \mid s_{N}, t\right) P_{\mathrm{eq}, \mu}\left(s_{N}\right),
$$

where $P\left(s_{N}^{\prime} \mid s_{N}, t\right)$ is the conditional probability for reaching the configuration $s_{N}^{\prime}$ in time $t$ starting from $s_{N}$. The correlation function may be expressed as

$$
\begin{aligned}
\left\langle A^{z}(t) B^{z}\right\rangle_{\mathrm{eq}, \mu}= & \frac{1}{2}(1+\mu)\left\langle 1_{+}\left|A^{z} \mathrm{e}^{\Psi t} B^{z}\right| P_{\mathrm{eq},+}\right\rangle \\
& +\frac{1}{2}(1-\mu)\left\langle 1_{-}\left|A^{z} \mathrm{e}^{W t} B^{z}\right| P_{\mathrm{eq},-}\right\rangle \\
= & \sum_{\dot{\Sigma}} \frac{1}{2}(1 \pm \mu)\left(Z_{N \pm} \mid Z_{N}\right)\left\langle 0_{ \pm}\left|A^{z} \mathrm{e}^{W(\beta) t} B^{z}\right| 0_{ \pm}\right\rangle
\end{aligned}
$$


In particular one finds for two Fourier components of the magnetization, using (5.15), (5.18) and (4.51)

$$
\begin{aligned}
\left\langle\sigma^{z}(q, t) \sigma^{z}\left(q^{\prime}\right)\right\rangle_{\mathrm{eq}, \mu}= & \frac{1}{2}(1+\mu)\left(Z_{N-} \mid Z_{N+}\right) \delta\left(q+q^{\prime}\right) N^{-1} \\
& \times \sum_{\substack{a_{1}<\mathbf{a}_{2} \\
\text { even }}} \delta\left(q_{1}+q_{2}-q\right) S\left(q_{1}, q_{2}, t\right) \\
& +\frac{1}{2}(1-\mu)\left(Z_{N+} / Z_{N-}\right) \delta\left(q+q^{\prime}\right) N^{-1} \\
& \times \sum_{\substack{q_{1}<q_{2} \\
\text { odd }}} \delta\left(q_{1}+q_{2}-q\right) S\left(q_{1}, q_{2}, t\right),
\end{aligned}
$$

where

$$
S\left(q_{1}, q_{2}, t\right)=\left|\mathrm{e}^{\mathrm{i} q_{1}}-\mathrm{e}^{\mathrm{i} q_{2}}\right|^{2} g_{a_{1}}^{2} g_{a_{2}}^{2} \exp \left[-\Lambda\left(q_{1}, q_{2}\right) t\right] .
$$

It can be shown that in the thermodynamic limit $N \rightarrow \infty$ and for $\mu=0$ this result is in agreement with that derived in ref. 6, eq. (7.10).

As a second example we consider the time-dependent average of a Fourier component of the energy density, corresponding to the operator

$$
E^{z}(q)=-J N^{-\frac{1}{2}} \sum_{j=1}^{N} \sigma_{j}^{z} \sigma_{j+1}^{z} \mathrm{e}^{-\mathrm{i} q j}
$$

Eliminating $\sigma_{1}^{z}$ and applying the $\sigma \tau$ transformation one finds

$$
E_{ \pm}^{z}(q)=-J N^{-\frac{1}{2}}\left[\sum_{j=2}^{N-1} \tau_{j-1}^{z} \tau_{j+1}^{z} \pm \tau_{2}^{z} \tau_{N}^{z} \mathrm{e}^{-\mathrm{i} q} \pm \tau_{N-1}^{z} \tau_{1}^{z} \mathrm{e}^{-\mathrm{i} q N}\right] .
$$

Fourier-transforming one finds for both even or odd $q$

$$
\begin{aligned}
& F_{ \pm}^{z}(q)=-J N^{-\frac{1}{2}} \sum_{q_{1}<q_{2}} \delta\left(q+q_{1}+q_{2}\right) \\
& \times\left[\mathrm{e}^{\mathrm{i} q_{1}-\mathrm{i} q_{2}}-\mathrm{e}^{-\mathrm{i} q_{1}+\mathrm{i} q_{2}}\right] T_{1}^{z}\left(q_{1}, q_{2} ; 0\right) \quad\left(q_{1}, q_{2}\right)_{\text {even }}^{\text {odd }} .
\end{aligned}
$$

Applying the transformation (4.42) and using (4.51) one finds that the only nonvanishing matrix elements (5.10) are

$$
\begin{aligned}
\left\langle 0_{ \pm}\left|E_{ \pm}^{z}(q)\right| q_{1}, q_{2}\right\rangle= & -J N^{-\frac{1}{2}}\left(Z_{N} / Z_{N \pm}\right) \delta\left(q-q_{1}-q_{2}\right) \\
& \times\left[\mathrm{e}^{-\mathrm{i} q_{1}+\mathrm{i} q_{2}}-\mathrm{e}^{\mathrm{i} q_{1}-\mathrm{i} q_{2}}\right] g_{q_{1}} g_{q_{2}} \quad\left(q_{1}, q_{2}\right) \begin{array}{c}
\text { odd } \\
\text { even }
\end{array} \\
\left\langle 0_{ \pm}\left|E_{ \pm}^{z}(q)\right| 0_{ \pm}\right\rangle=- & 2 J N^{-\frac{1}{2}}\left(Z_{N} / Z_{N \pm}\right) \delta(q) \sum_{q_{1}} f_{q_{1}} \sin 2 q_{1} \\
= & N^{-\frac{1}{2}} \delta(q)\left\langle\mathscr{H}_{N}\right\rangle_{\mathrm{eq}, \pm},
\end{aligned}
$$


where the latter equality is shown most easily from (4.15). The time-dependent average is therefore given by

$$
\begin{aligned}
\left\langle E^{z}(q)\right\rangle_{t}= & \left\langle E^{z}(q)\right\rangle_{\text {eq. } \mu}-J N^{-\frac{1}{2}} \sum_{q_{1}<q_{2}} p\left(q_{1}, q_{2}\right) \delta\left(q-q_{1}-q_{2}\right) \\
& \times\left(\mathrm{e}^{-\mathrm{i} q_{1}+1 q_{2}}-\mathrm{e}^{i q_{1}-1 q_{2}}\right) g_{q_{1}} g_{q_{2}} \exp \left[-\Lambda\left(q_{1}, q_{2}\right) t\right] .
\end{aligned}
$$

Similarly the equilibrium timecorrelation function is given by

$$
\begin{aligned}
& \left\langle\delta E^{z}(q, t) \delta E^{z}\left(q^{\prime}\right)\right\rangle_{\mathrm{eq}, \mu} \\
& =\frac{1}{2}(1+\mu) \delta\left(q+q^{\prime}\right) J^{2} N^{-1} \sum_{\substack{q_{1}<q_{2} \\
\text { odd }}} \delta\left(q_{1}+q_{2}-q\right) E\left(q_{1}, q_{2}, t\right) \\
& \quad+\frac{1}{2}(1-\mu) \delta\left(q+q^{\prime}\right) J^{2} N^{-1} \sum_{\substack{\mathbf{q}_{1}<q_{2} \\
\text { even }}} \delta\left(q_{1}+q_{2}-q\right) E\left(q_{1}, q_{2}, t\right),
\end{aligned}
$$

where the notation $\delta E^{z}$ indicates that the equilibrium average has been subtracted and where

$$
E\left(q_{1}, q_{2}, t\right)=\left|\mathrm{e}^{-\mathrm{i} q_{1}+\mathrm{i} q_{2}}-\mathrm{e}^{\mathrm{i} q_{1}-\mathrm{i} q_{2}}\right|^{2} g_{q_{1}}^{2} g_{q_{2}}^{2} \exp \left[-\Lambda\left(q_{1}, q_{2}\right) t\right] .
$$

Again it can be shown that in the thermodynamic limit $N \rightarrow \infty$ and for $\mu=0$ this result agrees with that found in ref. 6, eq. (6.21).

In conclusion we note that in the course of our analysis we have also found the operators $A^{z}$ with a simple behaviour in time. The averages of the operators $T_{1}^{z}\left(q_{2 k}, \beta\right)$ and $T_{2}^{z}\left(q_{2 k}, \beta\right)$ for states of the appropriate $\sigma$ parity decay according to the exponential law exp $\left[-\Lambda\left(q_{2 k}\right) t\right]$. Each of these operators is a linear combination of products of $2 l \tau^{z}$ operators with $0 \leqslant l \leqslant k$. From (4.20) it follows that this implies a sum of products of $\sigma^{z}$ operators which number from 0 to $N-1$. If we compare this with the hierarchy of moment equations for the averages of such products of $\sigma^{z}$ operators we see that we have succeeded in finding particular solutions of these coupled equations with a purely exponential behaviour in time.

Acknowledgement. In performing this work one of the authors (H.J.H.) participated in the research program of the "Stichting voor Fundamenteel Onderzoek der Materie" (F.O.M.) with financial support from the "Nederlandse Organisatie voor Zuiver-Wetenschappelijk Onderzoek" (Z.W.O.). 


\section{REFERENCES}

1) Glauber, R.J., J. math. Phys. 4 (1963) 294.

2) See also Suzuki, M. and Kubo, R,, J. Phys. Soc. Japan 24 (1968) 51.

3) Yahata, H. and Suzuki, M., J. Phys. Soc. Japan 27 (1969) 1421; Yahata, H., J. Phys. Soc Japan 30 (1971) 657.

4) Bedeaux, D., Shuler, K.E. and Oppenheim, I., J. statist. Phys. 2 (1970) 1.

5) Felderhof, B.U., Rep. math. Phys. 1 (1971) 215, 2 (1971) 151.

6) Felderhof, B. U. and Suzuki, M., Physica 56 (1971) 43. 